\title{
Electrosynthesis of conducting polyanilines in biphasic media
}

\author{
P A K NEDUNGADI, SUNITA BAWEJA and K ZUTSHI \\ Department of Chemistry. University of Rajasthan. Jaipur 302 004, India
}

\begin{abstract}
The electrochemical polymerization of aniline has been studied in the biphasic media. One phase comprises a polar medium containing some added electrolytes and the other a non-polar solvent containing the monomer. Ferric chloride has been used as the supporting electrolyte to catalyse the process of electropolymerization. Polyaniline was obtained in moderate yields with the highest $R_{p}$ of $0.0237 \%$ per hour and a maximum polymerization efficiency of $61.65 \times 10^{-2}$ mol Faraday. The observations and experimental results suggest a cationic mechanism.
\end{abstract}

Keywords. Electrosynthesis; conducting polymers; aniline.

\section{Introduction}

The spin density and conductivity of polyaniline range from $10^{19}-10^{20} \mathrm{~g}^{-1}$ and $10^{-13}-10^{2} \Omega^{-1} \mathrm{~cm}^{-1}$ respectively (Jozefowicz et al 1969; Langer 1978). A literature survey provided no information on the polymerization of aniline in a biphasic medium. A pressed pellet of polyaniline, when placed between a mercury electrode and an aqueous $\mathrm{H}_{2} \mathrm{SO}_{4}$ solution, produces an open circuit potential which decreases as the $\mathrm{pH}$ of the solution increases (Jozefowicz et al 1969). These observations encouraged us to undertake the present experiment. To avoid difficulties arising in conventional single phase electrolysis which gives rise to blocking of electrode formation of poorly conducting solutions etc, a biphasic system was employed. This comprised a non-polar phase, a solution of monomer in non-polar solvent such as benzene/toluene and a polar solvent with added electrolytes. The polar phase being more conducting allows the passage of sufficient electrolytic current and serves as a medium for electrolysis while the non-polar phase containing the bulk of monomer traps most of the radical cations formed at electrodes and acts as the polymerization medium. To facilitate transfer of radical cation formed in the polar phase to the nonpolar phase, the two phases are kept in close contact with each other by constant and rigorous stirring. The non-polar phase consisted mostly of solution of aniline in toluene/benzene. The polar phase consisted of $\mathrm{FeCl}_{3}$ solution in $0.1 \mathrm{M}$ aq. $\mathrm{H}_{2} \mathrm{SO}_{4}$.

Salts of zinc acetate, zinc bromide, zinc chloride, ferric sulphate, ferric chloride and potassium acetate were employed as the supporting electrolytes.

\section{Experimental}

\subsection{Materials}

Aniline (Aldrich), sulphuric acid (Aldrich) benzene and toluene (Ranbaxy) were used without further purification. Anhydrous zinc chloride, zinc bromide, zinc acetate, ferric chloride and potassium acetate (all $\mathrm{BDH}$ reagents) were used without further purification. 


\subsection{Cell assembly}

The electrochemical polymerization was carried out in a single compartment cell without separating the cathode and anode. In reactions, where catholyte and anolyte were to be analysed separately a split cell was employed whose compartments were separated by a sintered glass disc or a porous diaphragm. Pt sheets (working area $1 \mathrm{~cm}^{2}$ ) were used as the cathode and anode.

\subsection{Electrolysis and work-up}

Electrolysis was carried out by varying (i) the current, (ii) the concentration of monomer and (iii) the solvent of non-polar phase and the supporting electrolyte one by one.

The electrolytic process was controlied by a regulated power supply (MPI model MP 1016A) with provision to measure both the current and voltage drop through the cell. The electrolytic cell was kept in a water bath and the assembly containing the cell and water bath was mounted on a magnetic stirrer to provide adequate stirring of the experimental solutions. $\mathrm{N}_{2}$ gas was bubbled for 30 minutes after filling the cell with reactants prior to electrolysis. Metallic depositions, which were found at the cathode during electrolysis, were reduced to a minimum by changing the polarity of electrodes after every hour. The polymer formed in non-polar phase was precipitated with excess of acidified methanol and dried in vacuum.

\section{Results}

Experiments showed that no polymerization took place in the absence of electrolytic current. The yield of the polymer yield depended upon factors such as current strength, concentration of electrolyte, time of electrolysis, temperature and monomer concentration. Electropolymerization was carried out in a nitrogen atmosphere although air or oxygen had no effect during polymerization. Addition of electrolyte helped in initiation of polymerization reaction in addition to considerable increase in the conductance of electrolytic medium. The polyaniline so formed appeared structurally similar to para-coupled octamers, emeraldine and nigraniline from the infrared spectra (Mohilner et al 1962; Langer 1978). Table 1 summarizes the result of electropolymerization with different electrolytes. The conductivity determined by

Table 1. Electropolymerization of aniline at $40 \pm 1 \mathrm{C}$ with different electrolytes.

\begin{tabular}{lcccc}
\hline Electrolyte & $\begin{array}{c}\text { Conc. of } \\
\text { electrolyte } \\
\text { mol/litre }\end{array}$ & $\begin{array}{c}\text { Conversion } \\
\%\end{array}$ & $R_{p}$ & $\begin{array}{c}\text { Polymerisation } \\
\text { efficiency }\left(\times 10^{-2}\right) \\
\text { mol/Faraday }\end{array}$ \\
\hline Ferric chloride & 1.0 & 0.0460 & 0.0230 & 46.24 \\
Zinc chloride & 1.2 & 0.0301 & 0.015 & 30.26 \\
Zinc bromide & 1.0 & 0.0360 & 0.0180 & 36.19 \\
Zinc acetate & 1.0 & 0.0225 & 0.0113 & 22.62 \\
Potassium acetate & 2.0 & 0.0206 & 0.0103 & 20.17 \\
\hline
\end{tabular}

Current passed, $20 \mathrm{~mA}$; concentration of aniline, $1.5 \mathrm{~mol} / \mathrm{l}$; time of electrolysis, $2 \mathrm{~h}$; volume of polar and non-polar solvents, $50 \mathrm{ml}$ each. 
using a classical two-probe technique displays a value in the range of $10^{-4}$ to $2 \Omega^{-1} \mathrm{~cm}^{-1}$.

$\mathrm{FeCl}_{3}$ was found to be most suitable in producing polymers of higher yield. Figure 1 depicts the effect of magnitude of electrolytic current on conversion. The conversion of monomer to polymer initially increases with increase of current passed but at higher current a constant value was attained. The course of reaction was studied by changing the concentration of electrolyte at two different current strengths: $10 \mathrm{~mA}$ and $20 \mathrm{~mA}$. In both cases, the rate of polymerization increased with increase in concentration of electrolyte but finally attained a constant value. These results are shown in figure 2. The percentage of conversion as a function of time of electrolysis was plotted. The conversion varied linearly with time as shown in figure 3 with low yields at initial stages. Figure 4 shows the polymer formation as a function of monomer concentration. From the figure it is evident that the polymer formation is insensitive beyond a certain monomer concentration and below it the

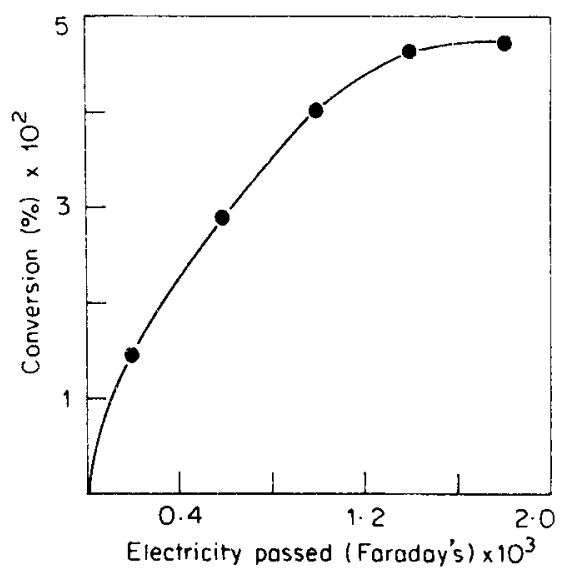

Figure 1. Percentage of conversion as a function of charge passed at $40 \pm 1^{\circ} \mathrm{C}$ in benzene.

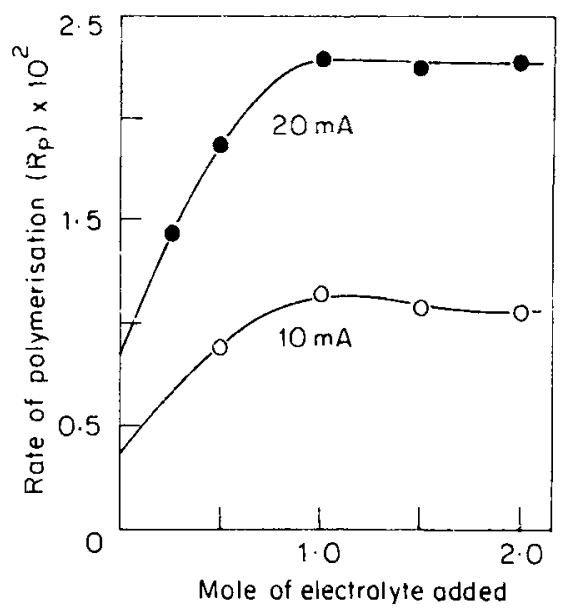

Figure 2. Rate of polymerization plotted against increasing concentration of electrolyte at $40 \pm 1{ }^{\circ} \mathrm{C}$ in benzene. 


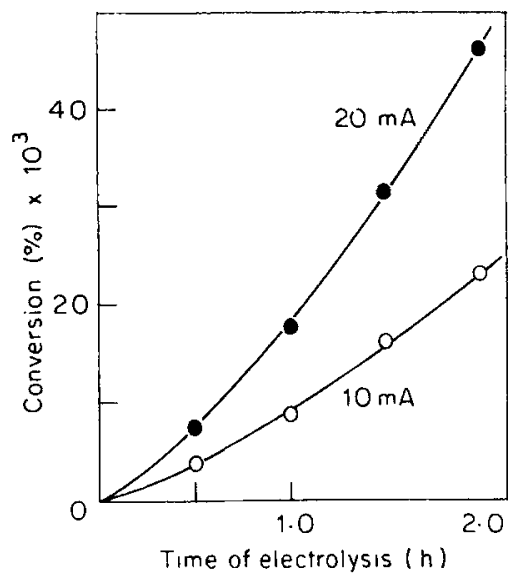

Figure 3. Percentage of conversion for electropolymerization of aniline as a function of time in benzene at $40 \pm 1 \mathrm{C}$.

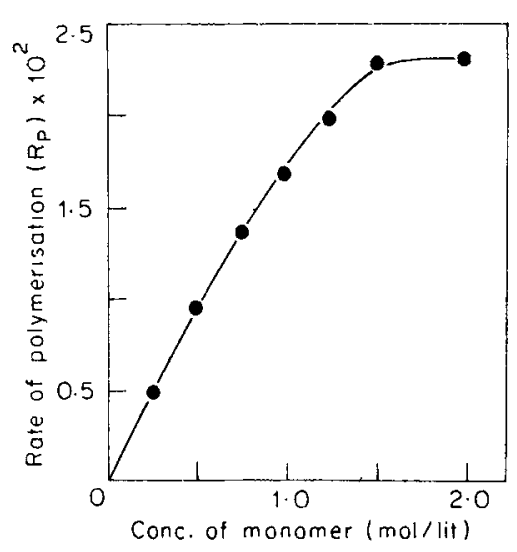

Figure 4. Rate of polymerization as a function of concentration of monomer in benzene at $40 \pm 1^{12} \mathrm{C}$.

yield is proportional to monomer concentration. The presence of air or hydroquinone, a radical quencher, had no effect on polymerization reaction whereas pyridine-cation quencher inhibits polymer formation.

Polyaniline was only slightly soluble in dimethyl formamide (DMF). The gel permeation chromatography of polyaniline in DMF + Sephadex column and the comparison of elution time for polyaniline and some other molecules showed that the soluble part of polyaniline had an average molecular weight of 80,000 (figure 5).

\section{Discussion}

After electrolysis when the two phases were allowed to separate the polar phase carried almost all the electrolyte and the non-polar phase carried the whole of the polymer. A free radical mechanism can be ruled out as there was no evidence of inhibiting effect of air or hydroquinone present in the system. Moreover, the 


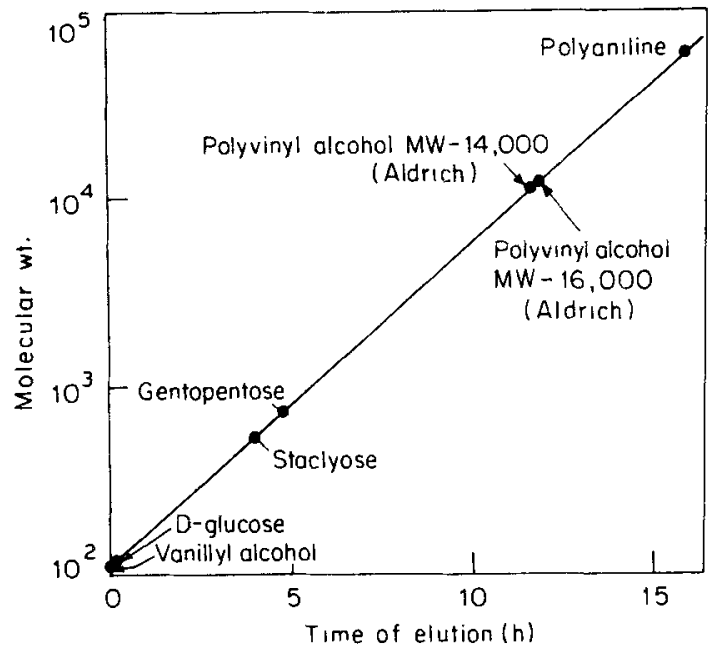

Figure 5. Comparison of elution time for polyaniline and other molecules of known molecular weight on the same column in DMF to compute the molecular weight.

inhibiting effect of pyridine, a cation quencher and the development of colour in the anode compartment (when split cell was employed) suggested cationic mechanism operating in this particular system. This was in sharp contrast to the radical mechanism observed for methyl methacrylate (Nayak and Bhakta 1983; Nedungadi et al 1987a), acrylonitrile (Nedungadi et al 1987b), acrylamide (Nedungadi et al $1987 \mathrm{c}$ ) and styrene under biphasic conditions.

As no polymerization took place in the absence of electrolyte and electrolytic current the following mechanism is suggested. Considering anode (Mohilner et al 1962; Bacon and Adams 1968; Sanyal et al 1985) to be the locus of polymerization, in the polar phase:

$$
\begin{aligned}
& 2 \mathrm{FeCl}_{3} \rightleftharpoons \mathrm{FeCl}_{4}^{-}+\mathrm{FeCl}_{2}^{+}, \\
& \mathrm{FeCl}_{4}^{-}-e^{-} \rightarrow \mathrm{FeCl}_{4} .
\end{aligned}
$$

The monomer acted as the $e^{-}$donor when $\mathrm{FeCl}_{4}$ thus formed moved to the interface. The coulombic forces of attraction start operating when $\mathrm{FeCl}_{4}^{-}$and monomer radical cation are held together (similar to ion pair formation) and the resulting species thereby propagate the polymerization process.

The overall scheme may be further extended as

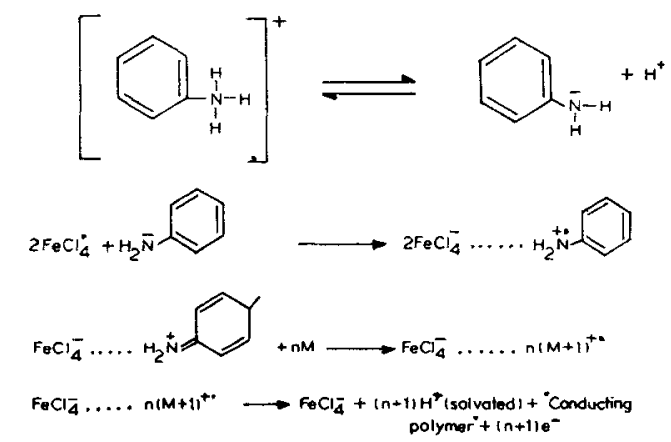


Only the monomer molecules lying very close to the reaction centre i.e. interface, can take part in polymerization reaction as the species $\mathrm{FeCl}_{4}^{-} \ldots \mathrm{H}_{2} \mathrm{~N} \bigcirc$ occurs only at the interface. The $\mathrm{FeCl}_{4}^{-}$part being held by the polar phase and the $\mathrm{H}_{2} \stackrel{+}{\mathrm{N}}$ part being held by the non-polar phase.

The formation of radical cation monomer is well substantiated because along with polyaniline some traces of 4-amino diphenylamine are also tound (head to tail coupling):

The mechanism put forward satisfactorily explains the increase in the rate of polymerization with increase in the concentration of the electrolyte, the increase in the yield of polymers with time of electrolysis and the increase in the rate of polymerization with increasing monomer concentration etc.

\section{Acknowledgement}

One of the author (PAKN) thanks CSIR, New Delhi for financial support.

\section{References}

Langer J 1978 Solid State Commun. 26839

Jozefowicz M, Yu L T, Perichon I and Buvet R 1969 J. Polym. Sci. C22 1187

Mohilner D M, Adams R N and Argersinger W J Jr 1962 J. Am. Chem. Soc. 842618

Nayak B and Bhakta R C $1983 \mathrm{~J}$ Appl. Electrochem. 13105

Nedungadi P A K, Gupta A, Mukerji S K and Zutshi K 1987a J. Electrochem. Soc. India 367

Nedungadi P A K, Gupta A, Taneja P and Zutshi K 1987b Bull. Electrochem. 3327

Nedungadi P A K, Gupta A, Mukerji S K and Zutshi K 1987c J. Electrochem. Soc. 3611

Sanyal S, Bhakta R C and Nayak B 1985 Macromolecules 181324

Bacon J and Adams R N 1968 J. Am. Chem. Soc. 906596 